\title{
Orienting to counterpredictive gaze and arrow cues
}

\author{
JASON TIPPLES \\ University of Hull, Hull, England
}

\begin{abstract}
In separate experiments, counterpredictive arrow, eye gaze, or abrupt-onset cues were used to test the hypothesis that individual differences in voluntary control influence involuntary orienting. In contrast with previous findings (Friesen, Ristic, \& Kingstone, 2004), involuntary orienting effects were found for arrow cues. Furthermore, for both eye gaze and arrow cues, individual differences in voluntary control were associated with involuntary orienting: Involuntary orienting effects were larger for participants who were more effective at using the cue to reorient attention, and also for participants who reported greater ability to control attention. Orienting to abrupt-onset cues was not correlated to individual differences in self-reported attentional control. The findings show that eye gaze and arrow cues instigate similar involuntary and voluntary effects and that involuntary orienting to symbolic cues is linked to individual differences in voluntary control.
\end{abstract}

What types of cues draw attention to a spatial location in an involuntary fashion? Until recently, research findings supported the distinction between involuntary, exogenous shifts of attention in response to peripherally presented cues (e.g., brief flashes of light) and voluntary, endogenous shifts in response to centrally presented cues (e.g., arrows). This distinction was blurred by findings from three independent laboratories (Driver et al., 1999; Friesen \& Kingstone, 1998; Langton \& Bruce, 1999), which showed that centrally presented gaze cues can also trigger in an involuntary orienting. Recently, these findings were extended (Friesen, Ristic, \& Kingstone, 2004). Eye gaze cues were found to produce orienting effects even when they were counterpredictive and it was beneficial for participants to redirect attention away from the cues. Furthermore, in support of the claim that eye gaze is a unique form of social attention cue, a further experiment (Friesen et al., 2004, Experiment 2) failed to record involuntary orienting to arrow cues. Recent research (Gibson \& Bryant, 2005; Vecera \& Rizzo, 2004, 2006) suggests that such findings may underestimate the influence of voluntary processes in the generation of involuntary orienting to symbolic cues. In the present research, a correlational approach was employed to reexamine the failure to find an involuntary orienting effect to arrow cues and to examine the relationship between involuntary and voluntary orienting to both arrow and gaze cues.

\section{Orienting to Counterpredictive \\ Gaze and Arrow Cues}

The counterpredictive orienting task used in recent research (Friesen et al., 2004) was designed to measure voluntary and involuntary orienting to gaze and arrow cues within the same task. Targets could appear in either a cued location (the same location as was indicated by the cue on $8 \%$ of trials), or a predicted location (opposite to the location indicated by the cue on $75 \%$ of trials), or in a location that was neither cued nor predicted (on a different axis to that indicated by the cue on the remaining $16 \%$ of trials). The neither predicted nor cued condition (NP-NC) acted as the new baseline. This type of baseline was considered an improvement over other baseline measures (e.g., the eyes staring straight ahead) because the cue stimuli on NP-NC trials were visually identical to the cues presented in other conditions. Responding in the cued condition was thought to indicate involuntary orienting because participants were told that the target rarely appeared in the same location as that indicated by the cue. Therefore, participants had no incentive to orient attention toward this location in comparison with the baseline condition. Conversely, responding in the predicted condition was thought to reflect voluntary processing because participants knew the target would most likely appear in the opposite location to the cue.

In addition to using a new baseline, the counterpredictive orienting task used by Friesen et al. (2004) included four stimulus onset asynchrony (SOA) conditions. These conditions were included to help investigate possible differences in the time course of involuntary and voluntary orienting to eye gaze cues. Cues were presented in short (105-msec), intermediate (600-msec), and two long (1,200-msec and 1,800-msec) SOA conditions. In keeping with predictions for involuntary orienting, responses were faster to targets in cued but not predicted locations in comparison with the baseline condition at the shortest (105-msec) SOA between cue and target. These effects continued up to $600 \mathrm{msec}$, where responses were faster 
to targets appearing in predicted locations in comparison with the new baseline. In other words, at an intermediate SOA, there was evidence for both reflexive and voluntary orienting to eye gaze. Furthermore, in the long SOA conditions $(1,200$ and $1,800 \mathrm{msec})$, voluntary orienting to the predicted location continued, but reflexive orienting to the cued location was eliminated. The extended time course for orienting on predicted but not cued trials might be taken as evidence of a slower acting, voluntary, controlled orienting mechanism. Overall, Friesen et al. interpret their findings as reflecting the operation of a two-process model of orienting to eye gaze, with relatively short-lived "reflexive" (involuntary) effects overlapping with, but eventually being replaced by, voluntary orienting effects.

\section{Is Involuntary Orienting Unique to Eye Gaze?}

A number of studies have shown that arrow cues also instigate involuntary shifts of attention (Hommel, Pratt, Colzato, \& Godijn, 2001; Ristic, Friesen, \& Kingstone, 2002; Tipples, 2002). In their second experiment, Friesen et al. (2004) examined whether arrow cues might also instigate involuntary shifts of attention. In a similar vein to eye gaze cues, faster responses were recorded on predicted than on NP-NC trials at an SOA of $600 \mathrm{msec}$ or greater. In contrast to the effects recorded for eye gaze cues, Friesen et al. failed to observe faster responses on cued trials than on NP-NC trials across all SOAs. However, it is important to note that in the $105-\mathrm{msec}$ SOA condition, responses were faster (albeit nonsignificantly) on cued trials $(377 \mathrm{msec}$ ) relative to $\mathrm{NP}-\mathrm{NC}$ trials $(385 \mathrm{msec})$. On the basis of their findings, Friesen et al. suggested that in comparison with eye gaze, orienting to arrow cues may be less involuntary and more susceptible to voluntary controlled processing.

\section{Are Involuntary and Voluntary Orienting Separable Processes?}

Do the effects recorded by Friesen et al. (2004, Experiment 1) reflect the operation of separable voluntary and involuntary mechanisms? Recent evidence (Gibson \& Bryant, 2005; Vecera \& Rizzo, 2006) appears to contradict this idea. First, on the basis of evidence from a patient (E.V.R.) with frontal lobe damage, Vecera and Rizzo (2004, 2006) suggest that orienting to eye gaze and arrow cues depends on voluntary, rather than reflexive, involuntary control. Previous research (Eslinger \& Damasio, 1985) had shown that E.V.R. was impaired at performing goal-directed, voluntary behaviors, despite demonstrating unimpaired executive functioning and working memory performance. Vecera and Rizzo (2004) reasoned that if orienting to symbolic cues depends on reflexive processes, then E.V.R. would orient attention to this type of cue. In contrast, if orienting to symbolic cues depended on voluntary process, then E.V.R.'s performance would be impaired. Vecera and Rizzo (2004) compared E.V.R.'s ability to orient attention to nonpredictive peripheral (exogenous) cues with his ability to orient to centrally presented, symbolic cues that were either predictive (Vecera \& Rizzo, 2004) or, in a later study (Vecera \& Rizzo, 2006), nonpredictive (uninformative). The peripheral cues induced the typical orienting effect (faster responses on valid than on invalid trials). However, E.V.R. did not appear to orient attention to either predictive or nonpredictive symbolic cues. In short, brain damage in the region associated with the voluntary control of attention led to a deficit in orienting to symbolic cues. This finding led Vecera and Rizzo $(2004,2006)$ to argue that orienting to symbolic cues depends on voluntary rather than reflexive processing, whereas reflexive orienting to peripheral cues does not depend on voluntary control.

The conclusion that orienting to peripheral (abruptonset) cues does not depend on voluntary control may be too strong, because other research has shown that the ability of abrupt-onset cues to trigger orienting is contingent on the perceiver's adopting specific attentional control settings (e.g., Folk \& Remington, 1998; Folk, Remington, \& Johnston, 1992). Specifically, increased similarity between cue and target increases the likelihood that abrupt-onset cues will trigger orienting. Furthermore, there is some evidence (Spence \& Driver, 1994, Experiment 6) that voluntary control may reduce orienting to peripheral cues when participants are actively encouraged to counteract the effects of such cues. Nevertheless, the conclusion reached by Vecera and Rizzo that voluntary control modulates orienting to symbolic cues still holds and, furthermore, is supported by additional evidence.

Findings from a recent study by Gibson and Bryant (2005) can be interpreted as showing that strategy-based processes influence involuntary orienting to symbolic cues. Gibson and Bryant designed a study to investigate methodological differences between an early study (Jonides, 1981), which failed to record involuntary orienting to uninformative symbolic cues, and later research (e.g., Hommel et al., 2001; Ristic et al., 2002; Tipples, 2002), in which there was reliable evidence for involuntary orienting. The authors noted that in the early study, arrow cues were presented for a brief duration $(25 \mathrm{msec})$ and were separated by a brief SOA between the onset of the cue and target (cue-to-target SOA). In contrast, later studies had tended to present symbolic cues for a longer duration and a longer cue-to-target SOA. To examine the effect of such differences, Gibson and Bryant varied cue duration and cue-to-target SOA. In the first experiment, the cue validity effect grew with increases in cue duration and cue-to-target SOA. More specifically, the effects were in keeping with both early research - with weak, nonsignificant cue validity effects at short cue durations and cue-to-target SOAs - and later research - with stronger significant effects at longer cue durations and cue-totarget SOAs.

In further experiments, Gibson and Bryant (2005) probed possible reasons for variations in the magnitude of the orienting effect with cue duration and cue-to-target SOA. In Experiment 2, cue duration was randomly intermixed within a block rather than varied in separate blocks, as had been the case in Experiment 1. Gibson and Bryant reasoned that if their initial findings were due to stimulus-based effects, then the magnitude would not differ between blocked (Experiment 1) and intermixed (Experiment 2) conditions, because stimulus factors are 
identical across both conditions. However, if the pattern of findings reflected strategic differences between cue conditions, then intermixing cue durations $(25 \mathrm{msec}$ and $200 \mathrm{msec}$ ) would make such strategy harder and, consequently, any differences due to cue duration would be eliminated. The pattern of findings supported the latter hypothesis. The difference in the magnitude of the cue validity effect between the $25-\mathrm{msec}$ and $200-\mathrm{msec}$ cue durations reported in Experiment 1 was eliminated when cue duration was intermixed within a block. In a final experiment, the target stimuli were preceded by either a bar or an uninformative directional cue. Participants were instructed to perform the target task only if an arrow cue preceded the display. This manipulation was carried out to ensure consistent processing of the cue stimuli across cue durations and cue-to-target SOA conditions. The findings added support to the claim that the differences recorded in Experiment 1 due to cue duration and SOA were due to strategy. Specifically, the cue validity effects were large and statistically equal in magnitude across the cue duration and SOA conditions. In summary, differences due to cue duration and SOA were eliminated when strategic processing of the cue was made harder (Experiment 2) or tightly controlled (Experiment 3 ).

The research findings of Gibson and Bryant (2005) are provocative, because they show that deliberate processing of the cue stimuli modulates orienting to uninformative central cues. Can the findings of Gibson and Bryant help explain the failure to find involuntary orienting effects for counterpredictive arrows and eye gaze cues (at longer SOAs) reported in previous research (Friesen et al., 2004)? According to Gibson and Bryant, failure to ensure that participants adequately process cue stimuli may lead to weakened cue validity effects and subsequently misleading conclusions based on the absence of such effects. Was failure to process the cue stimuli the reason for the absence of counterpredictive orienting effects for arrow stimuli reported by Friesen et al.?

At first glance, this conclusion appears unlikely, because the counterpredictive cuing task encourages the processing of the cue stimuli by making it beneficial to use the cue to reorient attention. However, unlike the task used by Gibson and Bryant (2005), these conditions do not make it obligatory to use the cue to reorient attention. Strategies could differ between individuals. Some participants might consider reorienting too effortful, and subsequently decide not to attend to the cue. Other participants might decide to use the cue to reorient attention. If adequate processing of the cue enhances involuntary orienting effects (Gibson $\&$ Bryant, 2005), then there should be a positive correlation between the magnitude of voluntary and involuntary orienting effects. Specifically, attempts to voluntarily use the cue are predicted to lead to adequate processing of the cue and, subsequently, involuntary orienting. If such a correlation exists, then inferring separate processes from null effects found by analyzing group-averaged data in a specific condition (e.g., a lack of voluntary orienting at a short SOA) may not be justified because this does not take into account the extent to which individuals differ in use of the cue to reorient attention.
The experiments reported here were designed to test the hypothesis that voluntary use of symbolic (Experiments 1-3) and abrupt-onset (Experiment 4) cues modulates involuntary orienting, by examining whether involuntary and voluntary orienting effects are positively correlated. Experiments 2-4 add a further test of the hypothesis that individual differences in voluntary control are linked to involuntary orienting, by examining correlations between self-reported ability to control attention and involuntary orienting. The designs and procedures for all of the experiments were similar to those used in previous research (Friesen et al., 2004), except that two, rather than four, SOA conditions were used in each experiment. In Experiments 1 and 2, cue-to-target SOAs of $105 \mathrm{msec}$ and 1,200 $\mathrm{msec}$ were used. In Experiments 3 and 4, a cue-to-target SOA of $600 \mathrm{msec}$ replaced the $1,200-\mathrm{msec}$ SOA condition.

\section{EXPERIMENT 1}

Friesen et al. (2004) used a 600-msec cue-to-target SOA in which voluntary and involuntary orienting effects were predicted to co-occur for eye gaze cues. Experiment 1 was not specifically designed to investigate the co-occurrence (or overlap) of voluntary and involuntary orienting at a specific SOA, and therefore this SOA was not included. Instead, the aims were to establish whether (1) involuntary orienting exists for arrow cues and (2) individual differences in voluntary orienting are linked to an increase in involuntary orienting. An attempt to replicate involuntary orienting to arrow cues at an SOA of $105 \mathrm{msec}$ between cue and target is important because the effects recorded by Friesen et al. were in the predicted direction in this condition. Therefore, involuntary orienting to arrow cues might exist for the group of participants overall and, consequently, the conclusion that eye gaze and arrow cues produce qualitatively different behavioral effects (see Friesen et al., 2004) may be premature. In Experiments 1 and 2, an SOA of 1,200 msec was chosen because voluntary orienting effects reported by Friesen et al. were largest (19 msec, averaged across cue types) in this condition. Therefore, the 1,200-msec SOA condition was selected to maximize the probability of replicating the voluntary orienting effect.

\section{Method}

Participants. In Experiment 1, the participants were 30 psychology undergraduates from the University of Hull (22 females and 8 males; age range $18-43$; mean age 22 years) who took part to fulfill a course requirement. All the participants had normal or correctedto-normal vision.

Apparatus and Stimuli. The arrow stimuli $(\leftarrow \downarrow \rightarrow \uparrow)$ were from the same font set as those used in previous research (Tipples, 2002, Experiment 1). Each arrow cue was preceded by a fixation star (*). The fixation star and cue stimuli appeared in the center of the screen. The stimuli were presented against a white background, on a 17 -in. computer monitor $(1,024 \times 768,60 \mathrm{~Hz})$. The target letters ( $\mathrm{F}$ or $\mathrm{T}$ ) subtended a vertical visual angle of $0.5^{\circ}$ and a horizontal visual angle of $0.5^{\circ}$. These letters were presented at a visual angle of $7.6^{\circ}$ to the left, to the right, above, or below the center of the fixation star. The letters were centered on either the vertical or the horizontal meridian. The arrow cues pointed directly at the center of the target stimuli. Stimulus presentation and data collection were controlled by 
E-Prime software (Schneider, Eschman, \& Zuccolotto, 2002) running on a $1-\mathrm{GHz}$ Pentium computer.

Design and Procedure. The trial sequence and design were similar to those used by Friesen et al. (2004) There were two main blocks of trials, each containing 224 trials split into balanced combinations of SOA $(105,1,200 \mathrm{msec})$, cue direction (left, right, up, down) and target type $(\mathrm{F}, \mathrm{T})$. For each of these combinations, there were four trial types (predicted, cued, NP-NC, catch). A target was not presented on catch trials. Catch trials accounted for approximately $7 \%$ of the total number of trials. Within the remaining trial types, targets appeared in either the opposite (predicted) location to that indicated by the cue (on $75 \%$ of trials), or the same (cued) location as that indicated by the cue (on $8 \%$ of trials), or in a location that was neither predicted nor cued (NP-NC) on the remaining trials $(8 \%$ clockwise and $8 \%$ counterclockwise, with respect to the cue direction). The main experimental trials were preceded by 20 practice trials, selected at random from the same combination of 224 possible trial types used in each main block of the experiment. The trials were presented in a new random order for each participant.

Before starting the experiment, participants were provided with written and verbal instructions. Participants were instructed to press the space bar with the index finger of their dominant hand as soon as the target letter appeared. They were instructed not to respond if a target letter failed to appear. They were told that targets appeared in the opposite location to that indicated by either cue type on $75 \%$ of trials.

All trials began with the fixation star. Participants were requested to fix their eyes on the center of the star and were also encouraged to keep their eyes focused on the arrow throughout each trial. In addition, a chinrest was used to minimize head movements. The viewing distance was $50 \mathrm{~cm}$. After $675 \mathrm{msec}$, the star was replaced by an arrow cue, pointing left, right, up, or down. Following an SOA of either 105 or $1,200 \mathrm{msec}$, a target letter (equally often an F or a T) appeared in one of the four locations until either a response was made or $1,500 \mathrm{msec}$ had elapsed. Catch trials were identical except that the target was not presented. Participants were requested to press the space bar as quickly as possible when the letter appeared, while keeping their eyes fixated on the arrow. The word "correct" (written in blue) followed trials on which participants correctly withheld responses (on catch trials) or pressed the space bar before the target was removed $(1,500 \mathrm{msec})$. The word "incorrect" (written in red) was presented either if participants responded on catch trials or if they failed to respond within 1,500 msec. Feedback was presented $500 \mathrm{msec}$ before a new trial began with the presentation of a fixation stimulus.

\section{Results}

Anticipations and outliers were defined as reaction times (RTs) less than $100 \mathrm{msec}$ and greater than $1,000 \mathrm{msec}$, respectively. False alarms were defined as responses on catch trials. Anticipations $(<1 \%$ of the total), false alarms $(<1 \%)$, and RT outliers $(<1 \%)$ were removed using these criteria. The means and standard errors of the RTs as a function of trial type (predicted, cued, NP-NC), and SOA (105 msec, 1,200 msec) are displayed in Figure 1. The means and standard deviations of the RTs can be found in Table 1.

Correlational analyses. ${ }^{1}$ Indices of involuntary effect were calculated for each individual, for each SOA separately, by subtracting the mean RT on cued trials from the mean RT on NP-NC trials. Similarly, to calculate indices of the voluntary orienting effect, the mean RT on predicted trials was subtracted from the mean RT on NP-NC trials. These indices were then divided by the overall mean RT to reduce the influence of overall mean RT. Finally, Pearson's product-moment correlation coefficients (Pearson's $r$ ) were calculated to examine the relationship between the indices of involuntary and voluntary orienting, at each SOA, separately. In support of the predictions, there was

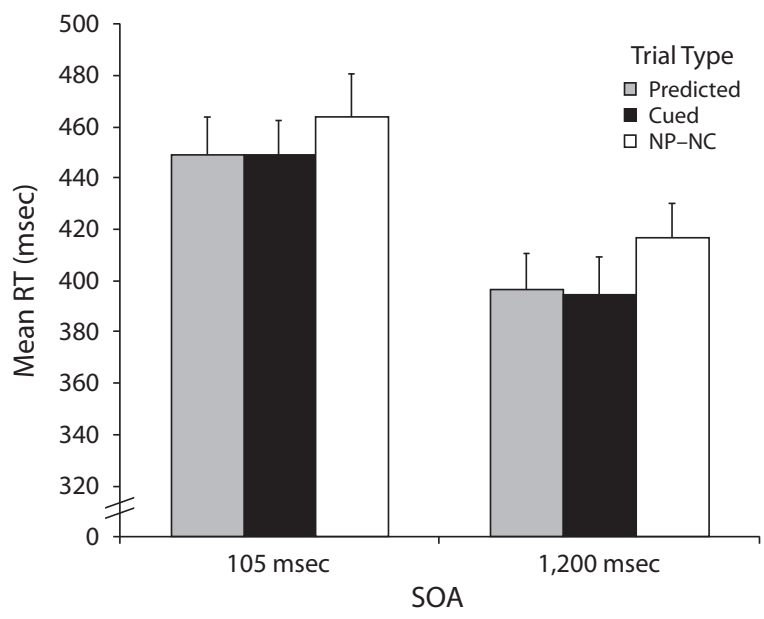

Figure 1. For the data gathered in Experiment 1 (arrow cues), the mean RTs with standard error bars as a function of trial type (predicted, cued, neither predicted nor cued [NP-NC]) and SOA (105 msec, 1,200 msec).

a significant positive correlation between the involuntary and voluntary indices in the 105-msec SOA condition $(r=$ $.48, p<.01)$ and the $1,200-\mathrm{msec}$ SOA condition $(r=.39$, $p<.05)$. Involuntary orienting grew in magnitude with increases in voluntary orienting.

Group-averaged data. Planned contrasts, in the form of single degree of freedom repeated measures $F$ tests, were conducted to test for involuntary and voluntary orienting, at each SOA, separately. ${ }^{2}$ For the pattern of data to indicate strongly involuntary orienting, faster responses were expected on cued than on NP-NC trials. In contrast to the findings of Friesen et al. (2004), this pattern emerged in the 1,200-msec SOA condition $[F(1,29)=14.50, p<$ $\left..001, \eta^{2}=.33\right]$. Although the pattern was in the same direction in the 105-msec SOA condition (see Figure 1), the effect failed to reach statistical significance $[F(1,29)=$ $\left.2.50, p=.12, \eta^{2}=.08\right]$. For voluntary orienting, faster responses were expected on predicted than on NP-NC trials. This was the case in both the $105-\mathrm{msec}$ SOA condition $\left[F(1,29)=10.42, p<.005, \eta^{2}=.26\right]$ and the $1,200-\mathrm{msec}$ SOA condition $\left[F(1,29)=23.19, p<.001, \eta^{2}=.44\right]$.

\section{Discussion}

In Experiment 1, involuntary orienting effects were largest for participants who were more effective at using the

Table 1

Mean Reaction Times (in Milliseconds) and Standard

Deviations for Experiment 1: Arrow Cues $(N=30)$

\begin{tabular}{|c|c|c|c|c|}
\hline \multirow[b]{3}{*}{ Trial Type } & \multicolumn{4}{|c|}{ Reaction Time } \\
\hline & \multicolumn{2}{|c|}{ 105-msec SOA } & \multicolumn{2}{|c|}{$1,200-\mathrm{msec} \mathrm{SOA}$} \\
\hline & $M$ & $S D$ & $M$ & $S D$ \\
\hline Predicted & 450 & 74 & 397 & 52 \\
\hline Cued & 449 & 80 & 395 & 54 \\
\hline $\mathrm{NP}-\mathrm{NC}$ & 464 & 79 & 417 & 56 \\
\hline
\end{tabular}

Note-SOA, stimulus onset asynchrony; NP-NC, neither predicted nor cued. 
arrow cue to reorient attention. Specifically, involuntary and voluntary orienting effects were correlated in both the 105-msec and 1,200-msec SOA conditions. In contrast with previous null findings (Friesen et al., 2004), the correlational analyses show that involuntary orienting to counterpredictive arrow cues does occur for participants who more effectively use the cue to reorient attention. The pattern of findings from the group-averaged data also supports the claim that involuntary orienting can occur in response to counterpredictive arrow cues. Involuntary orienting was recorded for arrow cues at a long SOA between the onset of the cues and the onset of the target. Specifically, in the long SOA condition, responses were faster to targets on cued trials, where the arrow cue pointed toward an unlikely target location, in comparison with trials on which the target was neither predicted nor cued. In other words, arrows appeared to trigger involuntary orienting, whereas in previous research (Friesen et al., 2004), only voluntary orienting (on predicted trials) was recorded to arrow cues presented for an SOA of $600 \mathrm{msec}$ or greater.

In partial agreement with previous research (Friesen et al., 2004), the involuntary orienting effect failed to reach significance in the short SOA condition. However, as the correlational analyses show, this failure to find an involuntary orienting effect is misleading, because involuntary orienting effects vary systematically with voluntary orienting effects; participants who were more effective at using the cue in the short SOA condition showed stronger involuntary orienting effects. Overall, the findings suggest that involuntary orienting is not unique to eye gaze cues, and that for symbolic cues, involuntary and voluntary orienting are linked processes.

\section{EXPERIMENT 2}

The finding that voluntary and involuntary orienting effects are correlated is new. Experiment 2 was designed to examine whether such a relationship exists for orienting to eye gaze. In addition, Experiment 2 extends Experiment 1 by investigating a possible link between self-reported attentional control and involuntary orienting. The findings of Vecera and Rizzo (2006) can be interpreted as showing that ability to control attention is related to orienting to symbolic cues. The patient E.V.R. had chronically poor ability to control attention. Similarly, Gibson and Bryant (2005) found that voluntary control was linked to the magnitude of involuntary orienting effects. Also, Experiment 1 showed that individual differences in ability to voluntarily redirect attention within the experiment were correlated with involuntary orienting effects. This finding can be extended by studying the relationship between involuntary orienting and self-reported ability to control attention. If involuntary orienting is influenced by voluntary controlled processing, then increases in self-reported ability to control attention should correlate with the orienting effect recorded on cued trials. Participants high in self-reported attentional control should be more able to control attention and consequently process the cue. To test this prediction, participants in Experiment 2 completed a questionnaire designed to measure attentional control
(Derryberry \& Reed, 2001; cited in Derryberry \& Reed, 2002), and scores on this measure were subsequently correlated with the indices of involuntary orienting (NP-NC minus cued trials).

\section{Method}

Participants. In Experiment 2, participants were all undergraduates from the University of Hull who took part to fulfill a course requirement. One of the participants terminated the experiment before data collection had finished. The data from this participant were not included in the final sample. The remaining sample consisted of 19 females and 10 males (age range 18-34 years; mean age 20.24 years). All the participants had normal or corrected-to-normal vision.

Apparatus and Stimuli. The apparatus was identical to that used in Experiment 1. The cue displays consisted of faces drawn with the eyes looking left, right, up, or down. A face with the eyes removed was used as a fixation stimulus. The faces measured $6.8^{\circ}$ of horizontal and $8.4^{\circ}$ of vertical visual angle and were centered horizontally on the computer screen. The faces were placed along the vertical axis of the screen so that the eyes on the face were level with the center of the target stimuli. In other words, the eyes appeared to look directly at the target. An example of the stimuli used and the sequence of events of a typical predicted trial type are displayed in Figure 2.

Attentional Control Scale. The Attentional Control Scale (Derryberry \& Reed, 2001; cited in Derryberry \& Reed, 2002) is designed to measure general capacity for attentional control, with subscale items measuring ability to (1) focus attention (e.g., "When concentrating, I can focus attention so that I become unaware of what's going on in the room around me"), (2) shift attention between tasks (e.g., "It is easy for me to alternate between two tasks"), and (3) flexibly control thought (e.g., "I can become interested in a new topic really quickly when I need to"). Items are scored on a 4-point scale $(1=$ almost never, $2=$ sometimes, $3=$ often, $4=$ always $)$. Eleven of the scale items are worded negatively and were reversescored prior to data analyses. Validity for the construct "attentional control" comes from factor analytic research (Derryberry \& Reed, 2001; cited in Derryberry \& Reed, 2002) and correlations between self-reported attentional control and RT measures of attention (Derryberry \& Reed, 2002; Mathews, Yiend, \& Lawrence, 2004).

Design and Procedure. The design and procedure were identical to those used in Experiment 1. After the RT experiment, participants completed the Attentional Control Scale. The questionnaire items were presented on the computer screen in a new random order for each participant.

\section{Results}

Anticipations ( $<1 \%$ of the total), false alarms $(<1 \%)$, and RT outliers $(<1 \%)$ were removed using the same criterion adopted in Experiment 1. The means and standard errors of the RTs as a function of trial type (predicted, cued, NP-NC) and SOA (105 msec, 1,200 msec) are displayed in Figure 3. The means and standard deviations of the RTs can be found in Table 2.

Correlational analyses. If involuntary and voluntary orienting are associated, then the indices of involuntary and voluntary orienting (calculated in the same manner as in Experiment 1) should correlate in both the 105- and 1,200-msec SOA conditions. In support of this hypothesis, significant positive correlations were found for the relationship between the involuntary and voluntary orienting indices in the 105-msec SOA condition $(r=.72, p<.01)$ and between the indices in the 1,200-msec SOA condition $(r=.38, p<.05)$. If involuntary orienting is associated with increased ability to control attention, then the indices 


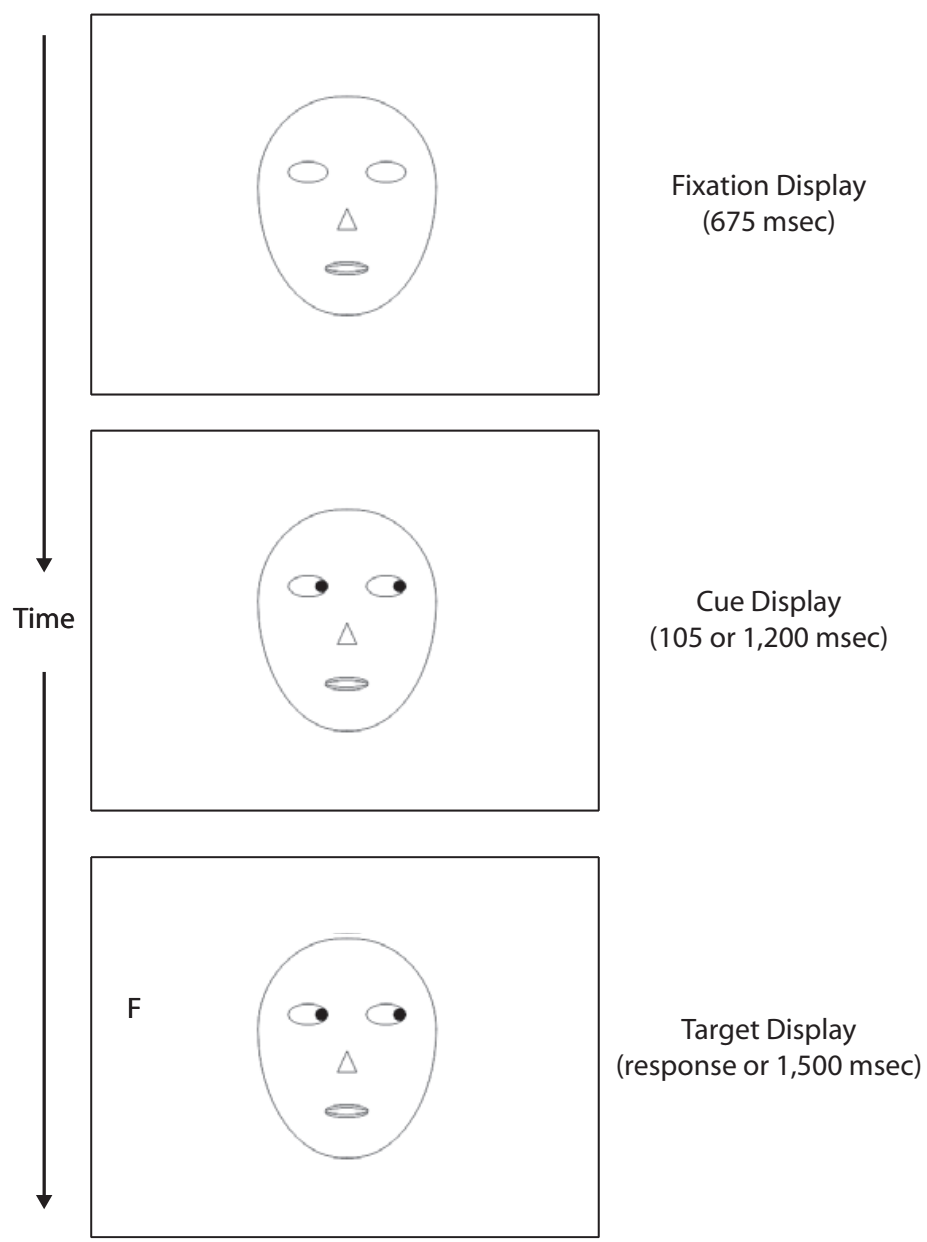

Figure 2. A typical (predicted) trial sequence used in Experiment 2. The trial sequence runs from top to bottom. After the presentation of a fixation stimulus for $675 \mathrm{msec}$, a cue was displayed for either 105 or $1,200 \mathrm{msec}$ before the target appeared either above, below, or to the left or right of the cue stimulus. The trial ended after the participant responded, or after $1,500 \mathrm{msec}$ had elapsed without a response. Feedback (not shown in Figure 2) was presented for $500 \mathrm{msec}$ at the end of each trial. This figure is not drawn to scale.

of involuntary orienting should correlate with attentional control scores. In partial support of this hypothesis, there was a significant positive correlation $(r=.55, p<.05)$ between the index of involuntary orienting and attentional control scores in the 105-msec SOA condition but not the $1,200-\mathrm{msec}$ SOA condition $(r=.11, p>.57)$. The correlation between attentional control and involuntary orienting in the short SOA condition remained significant after controlling for the correlation between the involuntary and voluntary index scores in the short SOA condition (partial $r=.52, p<.05$ ). All other correlations failed to reach significance (largest $r=.14, p>.5$ ).

Group-averaged data. Following Experiment 1, planned contrasts were used to test for involuntary and voluntary orienting effects. To reiterate, for the pattern of data to indicate involuntary orienting, faster responses were expected on cued than on NP-NC trials. As can be seen in Figure 3, this occurred in the $105-\mathrm{msec}$ SOA condition $[F(1,29)=$ $\left.16.70, p<.001, \eta^{2}=.37\right]$ but not in the 1,200-msec SOA condition $\left[F(1,29)=0.34, p=.56, \eta^{2}=.01\right]$. Second, for voluntary orienting, faster responses were expected on predicted than on NP-NC trials. This was the case in both the 105-msec SOA $\left[F(1,28)=7.09, p<.05, \eta^{2}=\right.$ $.20]$ and the $1,200-\mathrm{msec}$ SOA $[F(1,28)=9.13, p<.01$, $\left.\eta^{2}=.24\right]$ condition.

\section{Discussion}

Following Experiment 1, the findings from Experiment 2 support the hypothesis that voluntary, top-down control is linked to involuntary orienting to symbolic cues. In addition to replicating the positive association between voluntary and involuntary orienting effects reported in Experiment 1, in Experiment 2 there was a significant positive relationship between attentional control and involuntary orienting to eye gaze. Increased ability to control attention was associated with an increase in the magnitude of the in- 


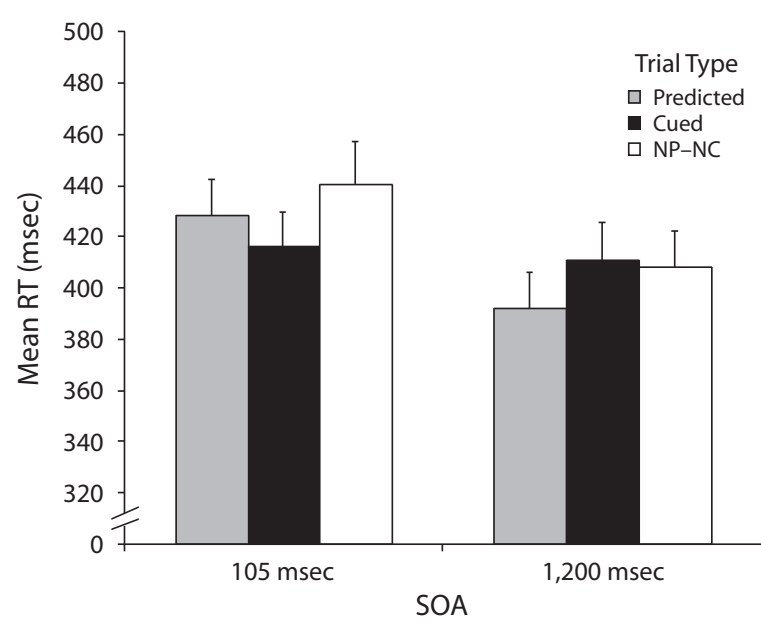

Figure 3. For the data gathered in Experiment 2 (eye gaze cues), the mean RTs with standard error bars as a function of trial type (predicted, cued, NP-NC) and SOA (105 msec, 1,200 msec).

voluntary orienting effect in the 105-msec SOA condition. In other words, participants who reported "good" attentional control were those that showed larger involuntary orienting effects. Again, this adds converging evidence to the findings from Experiment 1 and those reported elsewhere (Gibson \& Bryant, 2005; Vecera \& Rizzo, 2004, 2006) that voluntary controlled processes modulate involuntary orienting to symbolic cues.

With respect to the group-averaged data, the findings from Experiment 2 partly replicate those described in previous research (Friesen et al., 2004; Experiment 1) in a number of ways. First, eye gaze triggered orienting to unlikely target locations at an SOA of $105 \mathrm{msec}$. Second, this effect was absent at an SOA of 1,200 msec. Third, voluntary orienting was present in the 1,200 -msec SOA condition. However, in contrast to previous research, but in agreement with the data from Experiment 1 reported here, voluntary orienting (faster responses on predicted than on NP-NC trials) was present in the 105-msec SOA condition. Following Experiment 1, the latter finding shows that voluntary reorienting attention to symbolic cues can occur after only a short interval between cue and target. The pattern of data from Experiment 2 also shows that voluntary and involuntary orienting to eye gaze overlap in time. Although involuntary orienting was absent in the 1,200-msec SOA condition, voluntary and involuntary orienting were positively correlated across both SOA conditions. In other words, inferring "no involuntary orienting to eye gaze cues" in the 1,200 -msec SOA condition is misleading, because the lack of effects can be explained by individual differences in the voluntary use of the cue to reorient attention.

\section{EXPERIMENT 3}

The findings presented so far are limited: Only two SOA conditions have been used, whereas Friesen et al. (2004) used four, and it has yet to be established whether there exists a relationship between self-reported attentional control and involuntary orienting to arrow cues. Furthermore, the group-averaged, involuntary orienting effect to arrows in the 105-msec SOA condition was weak and failed to reach statistical significance. Therefore, a further study was run to (1) establish whether involuntary and voluntary orienting co-occur at the intermediate, 600-msec SOA used by Friesen et al. (2004), (2) test for a correlation between attentional control and involuntary orienting to arrow cues, and (3) attempt replication of the involuntary orienting effect to arrows in the $105-\mathrm{msec}$ SOA condition.

\section{Method}

In Experiment 3, the participants were 30 psychology undergraduates from the University of Hull (16 females and 14 males; age range 18-42 years; mean age 24 years) who took part to fulfill a course requirement. All of the participants had normal or corrected-to-normal vision. The design was the same as in Experiment 1 except that an SOA of 600 msec replaced the 1,200-msec SOA condition and (due to a programming error) participants undertook a single block of 224 trials. All other aspects of the stimuli and procedure were identical to those used in Experiment 1.

\section{Results and Discussion}

Following previous experiments, fewer than $1 \%$ anticipations, false alarms, and RT outliers were removed prior to data analyses. The means and standard deviations of the RTs can be found in Table 3. The means and standard errors of the RTs as a function of trial type (predicted, cued, $\mathrm{NP}-\mathrm{NC})$ and SOA (105 msec, $600 \mathrm{msec})$ are displayed in Figure 4.

Correlational analyses. The findings from Experiment 3 corroborate the idea that individual differences in voluntary control influence involuntary orienting to symbolic cues. Involuntary orienting effects grew with indi-

Table 2

Mean Reaction Times (in Milliseconds) and Standard

Deviations for Experiment 2: Eye Gaze Cues $(N=29)$

\begin{tabular}{|c|c|c|c|c|}
\hline \multirow[b]{3}{*}{ Trial Type } & \multicolumn{4}{|c|}{ Reaction Time } \\
\hline & \multicolumn{2}{|c|}{$105-\mathrm{msec} \mathrm{SOA}$} & \multicolumn{2}{|c|}{$1,200-\mathrm{msec} \mathrm{SOA}$} \\
\hline & $M$ & $S D$ & $M$ & $S D$ \\
\hline Predicted & 429 & 75 & 392 & 72 \\
\hline Cued & 416 & 72 & 411 & 77 \\
\hline $\mathrm{NP}-\mathrm{NC}$ & 440 & 90 & 409 & 73 \\
\hline
\end{tabular}

Note-SOA, stimulus onset asynchrony; NP-NC, neither predicted nor cued.

Table 3

Mean Reaction Times (in Milliseconds) and Standard Deviations for Experiment 3: Arrow Cues $(N=30)$

\begin{tabular}{lcccc}
\hline & \multicolumn{3}{c}{ Reaction Time } \\
\cline { 2 - 5 } Trial Type & $M$ & $S D$ & & \multicolumn{1}{c}{$1,200-\mathrm{msec}$ SOA } \\
\cline { 2 - 5 } \cline { 4 - 5 } & $M$ & 59 & 343 & 54 \\
\hline Predicted & 392 & 59 & 350 & 60 \\
Cued & 393 & 63 & 356 & 49 \\
NP-NC & 404 & S &
\end{tabular}

Note-SOA, stimulus onset asynchrony; $\mathrm{NP}-\mathrm{NC}$, neither predicted nor cued. 


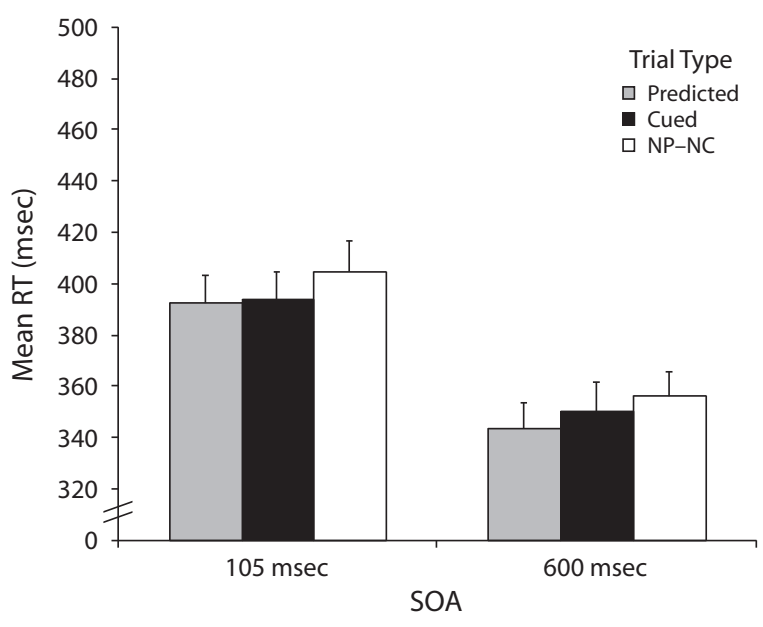

Figure 4. For the data gathered in Experiment 3 (arrow cues), the mean RTs with standard error bars as a function of trial type (predicted, cued, NP-NC) and SOA (105 msec, $600 \mathrm{msec})$.

vidual differences in both the magnitude of the voluntary orienting effect and separately, with self-reported attentional control. Specifically, the predicted positive relationship between the indices of voluntary and involuntary orienting was recorded in both the $105-\mathrm{msec}$ SOA condition $(r=.42, p<.05)$ and the $600-\mathrm{msec}$ SOA condition $(r=$ $.54, p<.005)$. The relationship between attentional control scores and the index of involuntary orienting was significant in the 600 -msec SOA condition $(r=.42, p<.05)$ but not the short SOA condition $(r=-.09, p=.61)$. The correlation between attentional control and involuntary orienting in the $600-\mathrm{msec}$ SOA condition remained significant after controlling for the correlation between the involuntary and voluntary index scores in the short SOA condition (partial $r=.37, p<.05$ ). All other correlations failed to reach significance (largest $r=.21, p>.2$ ).

Group-averaged data. The weak, nonsignificant involuntary orienting effect in the short SOA condition reported in Experiment 1 was now significant $[F(1,29)=$ $\left.6.28, p<.05, \eta^{2}=.18\right]$. However, the involuntary effect was weaker and consequently failed to reach significance in the $600-\mathrm{msec} \mathrm{SOA}$ condition $[F(1,29)=1.16, p=.29$, $\left.\eta^{2}=.04\right]$. There was a reliable voluntary orienting effect (NP-NC vs. predicted) in the $600-\mathrm{msec}$ SOA condition $\left[F(1,29)=7.23, p<.01, \eta^{2}=.20\right]$ and the $105-\mathrm{msec}$ SOA condition $\left[F(1,29)=16.24, p<.0001, \eta^{2}=.36\right]$.

\section{EXPERIMENT 4}

Experiments 1-3 support the claim that individual differences in voluntary control influence involuntary orienting to symbolic cues. However, it remains possible that the correlation between indices of voluntary and involuntary orienting might be best accounted for by the influence of a third variable. Similarly, the correlation between the indices of voluntary and involuntary orienting might be explained by common method variance. Therefore, it is important to show that this correlation disappears under theoretically relevant conditions that continue to share a common method. Abrupt-onset cues are one type of cue that might be less affected (under certain conditions) by individual differences in voluntary control. To reiterate, the findings of Vecera and Rizzo (2006) can be interpreted as showing that orienting to abrupt-onset cues is less affected by an individual's ability to control attention. Their patient showed intact orienting to peripherally presented abrupt-onset cues but did not orient attention to symbolic cues. Although the strong claim that orienting to abruptonset cues does not depend on voluntary control can be questioned on the basis of a number of findings (Folk et al., 1992; Folk \& Remington, 1998; Spence \& Driver, 1994), there is evidence that the effects of voluntary control on abrupt-onset cues might be limited under specific conditions. This evidence comes from a study (Spence \& Driver, 1994, Experiment 5) that did not examine individual differences but did use counterpredictive auditory abrupt-onset cues. This study showed that the ability to reorient attention (equivalent to the voluntary orienting effects reported here) increased as the cue-to-target SOA increased. At a short (100-msec) SOA between cue and target, participants were able to prevent orienting to counterpredictive abrupt-onset cues (involuntary effects were eliminated). However, reorienting effects did not emerge until a cue-to-target SOA of $400 \mathrm{msec}$. These findings suggest that voluntary control, and perhaps individual differences in voluntary control, might be limited at a short SOA between cue and target.

Experiment 4 was designed to test the hypothesis that orienting to abrupt-onset cues might be less influenced by individual differences in voluntary control. In Experiment 4 , an abrupt-onset cue appeared briefly $(50 \mathrm{msec})$ for either a short (105-msec) or long (600-msec) SOA between cue onset and target onset.

\section{Method}

Participants. In Experiment 4, there were 30 participants (20 females and 10 males; age range 18-45 years; mean age 22 years).

Stimuli. The abrupt-onset cue was a filled gray square measuring $1.06^{\circ} \times 1.06^{\circ}$ in size. To prevent lateral masking, the target and cue were spatially displaced so that the cue appeared nearer the fixation cross. Specifically, the distance from the outer edge of the abruptonset cue and the center of the target stimulus was $1.05^{\circ}$ of visual angle. In contrast to Experiments 1-3, the fixation star remained present throughout the trial. The abrupt-onset cue was presented for $50 \mathrm{msec}$ followed by a display (containing only the fixation star) for durations of either $55 \mathrm{msec}$ or $550 \mathrm{msec}$ to create the $105-\mathrm{msec}$ and 600 -msec SOA conditions, respectively. The instructions, number of trials, stimulus events, and all other elements of the method (including feedback and the administration of the questionnaire) were identical to those used in previous experiments.

\section{Results and Discussion}

Following previous experiments, fewer than $1 \%$ anticipations, false alarms, and RT outliers were removed prior to data analyses. The means and standard deviations of the RTs can be found in Table 4. The means and standard errors of the RTs as a function of trial type (predicted, cued, NP-NC) and SOA (105 msec, $600 \mathrm{msec}$ ) are displayed in Figure 5.

Correlational analyses. The results show that individual differences in voluntary control contribute less to invol- 
Table 4

Mean Reaction Times (in Milliseconds) and Standard Deviations for Experiment 4: Abrupt-Onset Cues $(N=30)$

\begin{tabular}{|c|c|c|c|c|}
\hline \multirow[b]{3}{*}{ Trial Type } & \multicolumn{4}{|c|}{ Reaction Time } \\
\hline & \multicolumn{2}{|c|}{$105-\mathrm{msec} \mathrm{SOA}$} & \multicolumn{2}{|c|}{$1,200-\mathrm{msec} \mathrm{SOA}$} \\
\hline & $M$ & $\overline{S D}$ & $M$ & $S D$ \\
\hline Predicted & 393 & 43 & 349 & 41 \\
\hline Cued & 438 & 56 & 443 & 50 \\
\hline $\mathrm{NP}-\mathrm{NC}$ & 397 & 43 & 366 & 50 \\
\hline
\end{tabular}

Note-SOA, stimulus onset asynchrony; NP-NC, neither predicted nor cued.

untary orienting to abrupt-onset cues. A positive relation between the indices of voluntary and involuntary orienting was recorded for the 600-msec SOA condition $(r=.48$, $p<.01)$ but not the $105-\mathrm{msec}$ SOA condition $(r=.26, p=$ .17). The relationship between attentional control scores and the index of involuntary orienting was not significant in either the $105-\mathrm{msec}$ SOA condition $(r=.05, p=.78)$ or the $600-\mathrm{msec}$ SOA condition $(r=-.02, p=.93)$.

Group-averaged data. In support of the hypothesis that participants are able to exert greater voluntary control as the SOA between the cue and target increases, the voluntary orienting effect was significant in the 600-msec SOA condition $\left[F(1,29)=19.98, p<.001, \eta^{2}=.40\right]$ but not the 105-msec SOA condition $[F(1,29)=1.58, p=$ $\left..22, \eta^{2}=.05\right]$. Similarly, inhibition in the form of slowing on cued in comparison with NP-NC trials grew in magnitude from the $105-\mathrm{msec}$ SOA condition $(M=41 \mathrm{msec})$ $\left[F(1,29)=26.68, p<.001, \eta^{2}=.48\right]$ to the $600-\mathrm{msec}$ SOA condition $(M=77 \mathrm{msec})[F(1,29)=75.17, p<$ $\left..001, \eta^{2}=.72\right]$. In summary, the findings can be interpreted as showing that orienting to abrupt-onset cues is less susceptible to the influence of individual differences at a short SOA between cue and target, and, moreover, the influence of voluntary control increases as the SOA increases between cue and target.

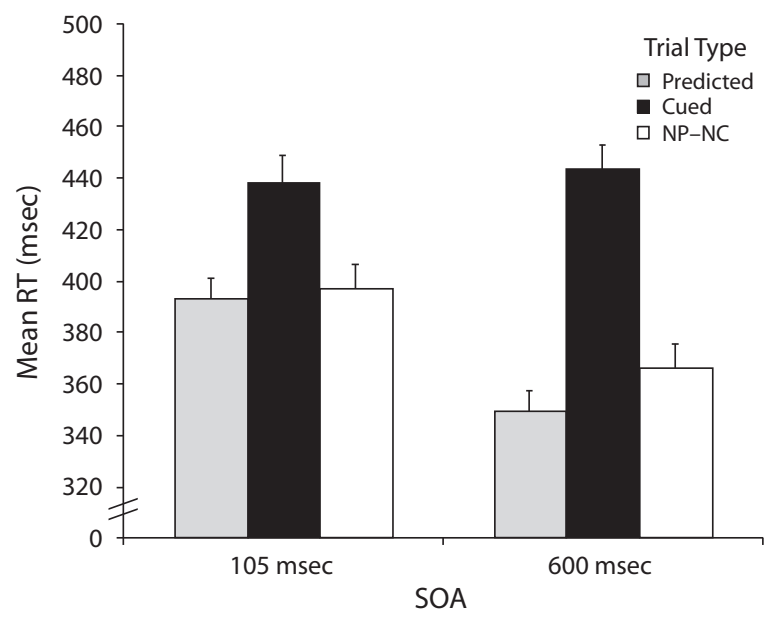

Figure 5. For the data gathered in Experiment 4 (abrupt-onset cues), the mean RTs with standard error bars as a function of trial type (predicted, cued, NP-NC) and SOA (105 msec, $600 \mathrm{msec})$.

\section{GENERAL DISCUSSION}

The present findings support the hypothesis that individual differences in the voluntary use of cues modulate involuntary orienting to symbolic cues. The pattern of correlations showed that involuntary orienting effects grew in magnitude with concomitant increases in voluntary orienting and self-reported voluntary control. The previous dissociation across SOA conditions (Friesen et al., 2004) can be explained by individual differences in voluntary control rather than separate involuntary and voluntary processes that have different but overlapping time courses. Specifically, in the present research, positive correlations between indices were recorded across SOA conditions despite nonsignificant group-averaged effects. The second conclusion is that involuntary orienting to counterpredictive cues is not unique to eye gaze cues. Involuntary orienting effects were consistently recorded for arrow cues in separate experiments, and, moreover, the effects of both eye gaze and arrow cues were reliably linked to individual differences in voluntary orienting and self-reported voluntary control. Finally, the effects due to symbolic cues were partially dissociable from those recorded for abrupt-onset cues. Involuntary orienting to abrupt-onset cues was not linked to individual differences in self-reported attentional control, and voluntary reorienting effects only emerged with time (in the 600-msec SOA condition).

One possible criticism of the present research is that the findings are correlational, and that correlational data do not imply causation. The effects could be explained in reverse: Involuntary orienting might cause larger voluntary orienting effects. However, it is important to note that such an explanation makes little theoretical sense in light of recent findings (Vecera \& Rizzo, 2004, 2006) suggesting that voluntary control is necessary for involuntary orienting effects and other findings (Gibson \& Bryant, 2005), whereby an experimental manipulation of voluntary control was shown to modulate involuntary orienting to symbolic cues. Furthermore, the correlation between attentional control and involuntary orienting is difficult to explain in reverse. Why would involuntary orienting lead to systematic increases in the ability to control attention? The term "involuntary" suggests that the process should be inflexible to voluntary control. Instead, the data suggest that orienting to symbolic cues is involuntary (in the sense that participants knew that cue direction was typically incorrect) but nevertheless linked to voluntary control processes. To reiterate, this reinforces the conclusion reached in other research (Gibson \& Bryant, 2005; Vecera \& Rizzo, 2004, 2006) that involuntary orienting is modulated by top-down processes. Indeed, one interpretation of the data described by Vecera and Rizzo $(2004,2006)$ is that attentional control is necessary for orienting to eye gaze.

Two further criticisms might be leveled at the correlational evidence. First, it is possible that a relationship with a third variable might best explain the relationship between voluntary control and involuntary orienting. Second, it could be argued that the correlation between indices reflects common method variance rather than a meaningful relationship between voluntary and invol- 
untary orienting. The correlation between self-reported attentional control and involuntary orienting goes some way toward addressing the issue of common method variance. These measures were correlated despite the fact that they are dissimilar, methodologically. Furthermore, the third variable problem and the common method variance explanation are also addressed by the pattern of findings recorded for abrupt-onset cues.

Individual differences in voluntary control were less influential with respect to involuntary orienting to abrupt-onset cues in a number of ways. First, the correlation between orienting to abrupt-onset cues and self-reported attentional control was not significant. Second, individual differences in voluntary orienting recorded during the experiment (the correlation between the indices) occurred only when the SOA between cue and target was sufficiently long. However, abrupt-onset cues were not impervious to voluntary control. The data corroborate previous findings (Spence \& Driver, 1994) by showing that voluntary control does affect orienting to abrupt-onset cues under certain conditions. Participants were able to counteract the effects of abruptonset cues although doing so led to a cost (inhibition). In addition, when given enough time (600 msec), participants were able to voluntarily reorient attention following abruptonset cues. The influence of voluntary control at the longer SOA was also evident in the form of increased inhibition at the cued location in the $600-\mathrm{msec}$ SOA condition than in the 105 -msec SOA condition. These findings follow previous research (Spence \& Driver, 1994) and show that the impact of voluntary control on orienting to abrupt-onset cues varies as a function of time. Given more time, participants are able to exert greater voluntary control over the effects of abrupt-onset cues.

In addition to the correlational evidence, findings at the group level (group-averaged data) add to the conclusion reached elsewhere (e.g., Hommel et al., 2001; Ristic et al., 2002; Tipples, 2002) that eye gaze and arrow cues produce qualitatively similar types of orienting. It is important to note that the argument proposed here is not that orienting to eye and arrow cues are identical - the group-averaged involuntary effects were absent for eye gaze cues but not for arrow cues in the 1,200-msec SOA condition. Instead, the argument is that counterpredictive gaze and arrows are similar in their ability to trigger involuntary orienting. Although the effects may vary between cue types in magnitude across SOA conditions, such differences do not justify the conclusion reached elsewhere (Friesen et al., 2004) that they produce qualitatively different effects, with orienting gaze cues but not arrows withstanding attempts at volitional control.

The group-averaged data are also informative with respect to the time course of orienting to symbolic and abrupt-onset cues. For symbolic cues, the data support the idea of a single process or interacting processes rather than separate involuntary and voluntary orienting processes that overlap at a specific time. Both voluntary and involuntary orienting effects occurred across SOA conditions. Again, although these effects varied between cue types, there was little evidence to suggest that involuntary orienting occurred at a specific SOA to the exclusion of voluntary orienting. Furthermore, even if an argument was made for separable processes on the basis of the present findings, the correlational data show that the interpretation of any null effects (e.g., involuntary orienting to arrows in the short SOA condition) should be made with caution, because individual differences in voluntary orienting exist, and these differences are linked to involuntary orienting effects.

\section{Individual Differences}

Analyses based on individual differences do have limitations: The causal status of the effects remained to be determined and, moreover, in the present research, individual differences cannot be used to explain all of the variance attributable to a specific effect. Nevertheless, consideration of such effects can provide useful converging evidence for a hypothesis (e.g., voluntary control modulates involuntary orienting). Moreover, other research has highlighted the value of considering individual differences in the study of attentional processes. For example, Derryberry and Reed (2002) found that attentional bias toward threat-related information and safety cues in anxiety was mediated by individual differences in attentional control. Clearly, the relationship between ability to control attention and other types of attentional processing warrants further investigation. More generally, investigating individual differences in orienting to eye gaze appears to be a profitable avenue for research. Recent research (Bayliss, di Pellegrino, \& Tipper, 2005) has shown that in comparison with women, men show reduced orienting to symbolic cues and, furthermore, increases in scores on the Autism Spectrum Quotient (Baron-Cohen, Wheelwright, Skinner, Martin, \& Clubley, 2001) are negatively correlated with the magnitude of orienting effects. Finally, other studies have shown that trait fearfulness (Tipples, 2006) and trait anxiety (Mathews, Fox, Yiend, \& Calder, 2003) potentiate orienting to eye gaze for faces displaying fear (see also Hietanen \& Leppänen, 2003).

\section{Conclusion}

The main conclusions are that (1) individual differences in voluntary control contribute to involuntary orienting effects and (2) eye gaze is not unique: Arrow cues also trigger involuntary orienting (Hommel et al., 2001; Ristic et al., 2002; Tipples, 2002). More broadly, the findings add to growing evidence (e.g., Folk \& Remington, 1998) that the deployment of attention is the product of interaction between voluntary and involuntary processes.

\section{AUTHOR NOTE}

Correspondence concerning this article should be addressed to J. Tipples, Department of Psychology, University of Hull, Hull, England, HU6 7RX (e-mail: j.tipples@hull.ac.uk).

\section{REFERENCES}

Baron-Cohen, S., Wheelwright, S., Skinner, R., Martin, J., \& Clubley, E. (2001). The autism-spectrum quotient (AQ): Evidence from Asperger syndrome/high-functioning autism, males and females, scientists and mathematicians. Journal of Autism \& Developmental Disorders, 31, 5-17.

Bayliss, A. P., Di Pellegrino, G., \& Tipper, S. P. (2005). Sex differences in eye gaze and symbolic cueing of attention. Quarterly Journal of Experimental Psychology, 58A, 631-650. 
DERryberRy, D., \& REed, M. A. (2001). Attentional control, trait anxiety, and the regulation of irrelevant response information. Manuscript submitted for publication.

Derryberry, D., \& Reed, M. A. (2002). Anxiety-related attentional biases and their regulation by attentional control. Journal of Abnormal Psychology, 111, 225-236.

Driver, J., Davis, G., Ricciardelli, P., Kidd, P., Maxwell, E., \& BARON-COHEN, S. (1999). Gaze perception triggers reflexive visuospatial orienting. Visual Cognition, 6, 509-540.

EsLinger, P. J., \& DAMASIO, A. R. (1985). Severe disturbance of higher cognition after bilateral frontal lobe ablation: Patient EVR. Neurology, 35, 1731-1741.

Folk, C. L., \& Remington, R. (1998). Selectivity in distraction by irrelevant featural singletons: Evidence for two forms of attentional capture. Journal of Experimental Psychology: Human Perception \& Performance, 24, 847-858.

Folk, C. L., Remington, R. W., \& Johnston, J. C. (1992). Involuntary covert orienting is contingent on attentional control settings. Journal of Experimental Psychology: Human Perception \& Performance, 18, 1030-1044.

Friesen, C. K., \& Kingstone, A. (1998). The eyes have it! Reflexive orienting is triggered by nonpredictive gaze. Psychonomic Bulletin \& Review, 5, 490-495.

Friesen, C. K., Ristic, J., \& Kingstone, A. (2004). Attentional effects of counterpredictive gaze and arrow cues. Journal of Experimental Psychology: Human Perception \& Performance, 30, 319-329.

Gibson, B. S., \& BRYANT, T. A. (2005). Variation in cue duration reveals top-down modulation of involuntary orienting to uninformative symbolic cues. Perception \& Psychophysics, 67, 749-758.

Hallahan, M., \& Rosenthal, R. (1996). Statistical power: Concepts, procedures, and applications. Behaviour Research \& Therapy, 34 489-499.

Hietanen, J. K., \& Leppänen, J. M. (2003). Does facial expression affect attention orienting by gaze direction cues? Journal of Experimental Psychology: Human Perception \& Performance, 29, 1228-1243.

Hommel, B., Pratt, J., Colzato, L., \& Godijn, R. (2001). Symbolic control of visual attention. Psychological Science, 12, 360-365.

JoNIDES, J. (1981). Voluntary versus automatic control over the mind's eye's movement. In J. [B.] Long \& A. [D.] Baddeley (Eds.), Attention and performance $I X$ (pp. 187-203). Hillsdale, NJ: Erlbaum.

Langton, S. R. H., \& BRUCE, V. (1999). Reflexive visual orienting in response to the social attention of others. Visual Cognition, 6, 541-567.
Mathews, A., Fox, E., Yiend, J., \& Calder, A. (2003). The face of fear: Effects of eye gaze and emotion on visual attention. Visual Cognition, 10, 823-835.

Mathews, A., Yiend, J., \& Lawrence, A. D. (2004). Individual differences in the modulation of fear-related brain activation by attentional control. Journal of Cognitive Neuroscience, 16, 1683-1694.

Ristic, J., Friesen, C. K., \& Kingstone, A. (2002). Are eyes special? It depends on how you look at it. Psychonomic Bulletin \& Review, 9 , 507-513.

Schneider, W., Eschman, A., \& Zuccolotto, A. (2002). E-Prime user's guide. Pittsburgh: Psychology Software Tools.

SPENCE, C. J., \& DRIVER, J. (1994). Covert spatial orienting in audition: Exogenous and endogenous mechanisms. Journal of Experimental Psychology: Human Perception \& Performance, 20, 555-574.

Tipples, J. (2002). Eye gaze is not unique: Automatic orienting in response to uninformative arrows. Psychonomic Bulletin \& Review, 9 , 314-318.

TiPPLES, J. (2006). Fear and fearfulness potentiate automatic orienting to eye gaze. Cognition \& Emotion, 20, 309-320.

VECERA, S. P., \& Rizzo, M. (2004). What are you looking at? Impaired "social attention" following frontal-lobe damage. Neuropsychologia, 42, 1647-1665.

Vecera, S. P., \& Rizzo, M. (2006). Eye gaze does not produce reflexive shifts of attention: Evidence from frontal-lobe damage. Neuropsychologia , 44, 150-159.

\section{NOTES}

1. An alternative way to conceptualize this effect is to create a criterion for selecting participants on the basis of whether they were deemed to have used the cue to reorient attention. For example, the involuntary orienting effect is significant in both the short and long SOA conditions (both $p \mathrm{~s}<.05$ ) for the 15 participants with the largest indices of voluntary orienting (averaged across SOA conditions), but not for those with the lowest indices of voluntary orienting $(p>.2)$.

2. Omnibus ANOVA was not used because it fails to test the critical effects for this research, and therefore lacks statistical power. For discussion of this issue and others relating to statistical power, see Hallahan and Rosenthal (1996).

(Manuscript received February 10, 2006; revision accepted for publication June 25, 2007.) 